\title{
Temperature dependence of the $\bar{E} \rightarrow 2 \bar{A}$ far-infrared absorption line of excited chromium ions in ruby
}

\author{
H. Lengfellner, J. Hummel, H. Netter, and K. F. Renk \\ Institut für Angewandte Physik, Universität Regensburg, 8400 Regensburg, Federal Republic of Germany
}

Received November 24, 1982

By using far-infrared laser spectroscopy we have studied the temperature dependence of the absorption that is due to transitions between the $\bar{E}\left({ }^{2} E\right)$ and $2 \bar{A}\left({ }^{2} E\right)$ levels of excited $\mathrm{Cr}^{3+}$ ions in ruby. From the temperature dependence of the linewidth we determined the inhomogeneous and the homogeneous contributions to line broadening. From the homogeneous width the lifetime of the $2 \bar{A}$ level was obtained. We found that, for temperatures up to $70 \mathrm{~K}$, the lifetime is due mainly to one-phonon relaxation, whereas at higher temperatures another relaxation process, probably a two-phonon Raman process, becomes strong.

The spin-phonon interaction between the excited states $\bar{E}\left({ }^{2} E\right)$ and $2 \bar{A}\left({ }^{2} E\right)$ of $\mathrm{Cr}^{3+}$ ions in ruby, which have an energy separation of $29 \mathrm{~cm}^{-1}$, has been the subject of various studies. Blume et al. ${ }^{1}$ reported an approximate calculation of the spin-lattice relaxation time $T_{1}\left(3 \times 10^{-10} \mathrm{sec}\right)$ for the $2 \bar{A} \rightarrow \bar{E}$ one-phonon relaxation, and Rives and Meltzer ${ }^{2}$ concluded from a fluorescence experiment that $T_{1}$ may be larger than the theoretical value by a factor of 3-4. We report on a spectroscopic study using a far-infrared technique that allows us to determine $T_{1}$ from the temperature dependence of the $\bar{E} \rightarrow 2 \bar{A}$ absorption line.

We point out that the spin-phonon interaction of $29-\mathrm{cm}^{-1}$ phonons with excited $\mathrm{Cr}^{3+}$ ions is of importance in phonon spectroscopy, in which phonons are detected by $\bar{E} \rightarrow 2 \bar{A}$ transitions and generated by $2 \bar{A}$ $\rightarrow \bar{E}$ spontaneous or stimulated relaxation processes and where resonance trapping of phonons occurs by subsequent $\bar{E} \leftrightarrow 2 \bar{A}$ emission and absorption processes. $^{3}$

The principle of our experiment is shown in Fig. 1a. As a source of far-infrared (FIR) radiation, a HCN laser emitting radiation at an energy of $29.7 \mathrm{~cm}^{-1}$ is used. In a magnetic field parallel to the crystalline $c$ axis, the $\bar{E}$ and $2 \bar{A}$ levels are split, and the FIR-laser radiation is resonant with respect to the $\bar{E}_{-} \rightarrow 2 \bar{A}_{+}$transition at a magnetic-field strength $B=0.5 \mathrm{~T}$ and to the $\bar{E}_{-} \rightarrow 2 \bar{A}_{-}$ transition at $B=2 \mathrm{~T}$. When the $\bar{E}$ levels are populated by optical excitation of the crystal, absorption of FIRlaser radiation occurs at these magnetic fields. By tuning the field, one can determine the shape and the width of the FIR absorption line. We have performed our experiment on a crystal $^{4}$ containing 0.05 wt $\%$ $\mathrm{Cr}_{2} \mathrm{O}_{3}$.

In an earlier experiment ${ }^{5}$ the same method was applied to determine the linewidth of the $\bar{E} \rightarrow 2 \bar{A} \mathrm{ab}$ sorption line for $\mathrm{Al}_{2} \mathrm{O}_{3}$ crystals that contained different concentrations of $\mathrm{Cr}_{2} \mathrm{O}_{3}$. In this experiment the sample was cooled by superfluid helium. For our new experiment we constructed a sample mount that allowed us to remove from the crystal at high temperature a large amount of heat that was caused by the optical pumping. Strong optical pumping was necessary because the FIR absorption cross section for the $\bar{E} \rightarrow 2 \bar{A}$ transitions is small. To reach a relatively large FIR absorption, we performed the experiment on a long crystal $(5 \mathrm{~mm} \times 5$ $\mathrm{mm} \times 15 \mathrm{~mm}$ ). The crystal was placed in the center of a superconducting magnet that had a large homogeneous field region. Part of the experimental arrangement is shown in Fig. 1b. The ruby crystal is mounted in a vacuum chamber that is surrounded by liquid helium. The crystal is in weak thermal contact with the cool walls of the chamber by means of a steel tube that can be heated electrically. With this arrangement the temperature of the optically irradiated crystal could be varied from $10 \mathrm{~K}$ to temperatures above $100 \mathrm{~K}$. The crystal temperature, which was higher than the temperature of the sample mount because of the optical

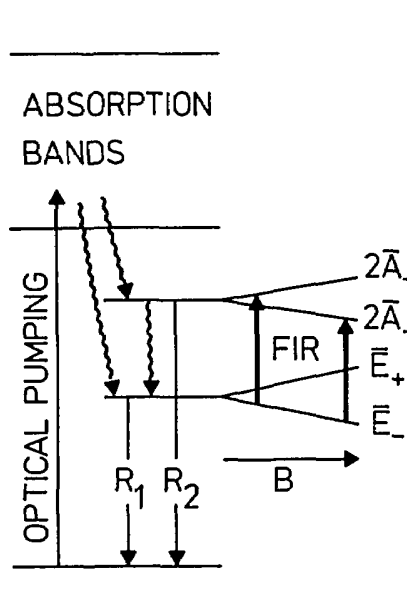

a

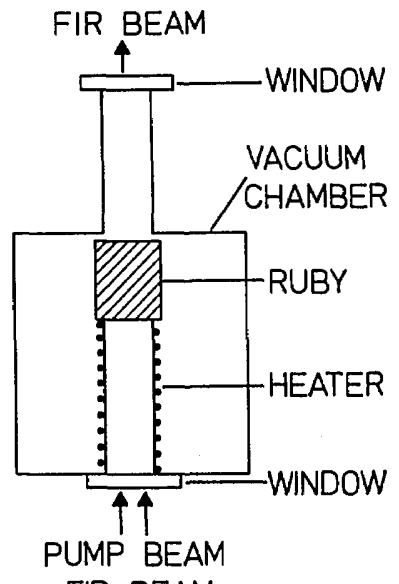

FIR BEAM
Fig. 1. a, Energy levels of $\mathrm{Cr}^{3+}$ ions in ruby and b, part of the experimental arrangement. 
pumping, was determined in a simple way by use of the fluorescence radiation. In an equilibrium the ratio of the populations of the $\bar{E}$ and $2 \bar{A}$ levels is given by a Boltzmann distribution, and the crystal temperature $T$ is therefore obtained from the relation $I_{2} / I_{1}=$ $\exp (-\Delta E / k T)$, where $I_{2} / I_{1}$ is the ratio of the fluorescence intensities of the $R_{2}$ and the $R_{1}$ lines, $\Delta E / h c=29$ $\mathrm{cm}^{-1}, k$ is Boltzmann's constant, $h$ is Planck's constant, and $c$ is the velocity of light. Fluorescence radiation is guided by fiber optics and separated spectrally by a double-grating monochromator. Temperature control was achieved by using a conventional temperature sensor (a diode) attached to the crystal mount.

Optical excitation of the crystal was achieved with chopped radiation of a krypton laser (with a power up to $2 \mathrm{~W}$ ). The FIR-laser radiation (power $10^{-2} \mathrm{~W}$ ) was focused on the ruby crystal and transmitted by a metal tube to a Golay detector. The Golay detector signal, analyzed by a lock-in technique, was proportional to the FIR absorption induced by the optical excitation.

Experimental signal curves showing the FIR absorption that is due to the $\bar{E}_{-} \rightarrow 2 \bar{A}_{+}$transition are drawn in Fig. 2. At low crystal temperature (see curve for $17 \mathrm{~K})$ the linewidth $\Delta B(209 \mathrm{G})$ corresponds to a linewidth in the frequency scale of $\Delta \nu=570 \mathrm{MHz}$ (FWHM). Here we used the relation $h \times \Delta \nu=1 / 2(g \bar{E}$ $\left.+g_{2 \bar{A}}\right) \times \Delta B \times \mu_{B}$, where $\mu_{B}$ is Bohr's magneton and $g_{\bar{E}}=2.445$ (Ref. 6) and $g_{2 \bar{A}}=1.47$ (Ref. 7) are the effective $g$ factors of the $\bar{E}$ and $2 \bar{A}$ levels, respectively.

At a crystal temperature of $60 \mathrm{~K}$ (lower curve in Fig. 2) the linewidth has doubled in comparison with the width at low temperature. The absorption at $60 \mathrm{~K}$ is much smaller than at $17 \mathrm{~K}$ because the $\bar{E}$ and $2 \bar{A}$ levels become more and more nearly equally populated with increasing temperature. The absorption curves in Fig. 2 are obtained at concentrations of excited $\mathrm{Cr}^{3+}$ ions in the $\bar{E}_{-}$state of about $3 \times 10^{17} \mathrm{~cm}^{-3}$. We estimate that the absorption cross section in the line maximum is $\sigma_{0}$ $\simeq 2 \times 10^{-19} \mathrm{~cm}^{2}$ at low temperature. The value of $\sigma_{0}$ decreases with increasing temperature, corresponding to the line broadening. We point out that we found no dependence of the linewidth on the strength of the optical excitation.

In addition to the experimental curves, Lorentzian and Gaussian line profiles are indicated in Fig. 2. At low temperatures, a line shape between a Lorentzian and a Gaussian profile is obtained, whereas at high temperature the line shape becomes almost Lorentzian.

We have also performed absorption measurements for the transition $\bar{E}_{-} \rightarrow 2 \bar{A}_{-}$at $2 \mathrm{~T}$. We found that this transition has the same oscillator strength as the $\bar{E}_{-} \rightarrow$ $2 \bar{A}_{+}$transition $^{5}$ and that the linewidths (in the frequency scale) are also the same. The experimental results of the linewidth measurements are summarized in Fig. 3. At low temperatures (up to $30 \mathrm{~K}$ ) the linewidth is almost constant, and it increases at higher temperatures. At our highest temperature $(100 \mathrm{~K})$ the width has increased by a factor of 4 compared with the value at low temperature.

Our experimental results suggest that the linewidth of the $\bar{E} \rightarrow 2 \bar{A}$ transition is due partially to homogeneous broadening and partially to inhomogeneous broadening. The line shape can be described well by a Voigt profile with the linewidth ${ }^{8}$

$$
\Delta \nu=\left(\Delta \nu_{L}^{2}+\Delta \nu_{G}^{2} / \ln 2\right)^{1 / 2},
$$

where $\Delta \nu_{L}$ and $\Delta \nu_{G}$ are the Lorentzian and Gaussian contributions to the actual width.

We attribute the Lorentzian contribution of the line broadening to lifetime broadening of the $2 \bar{A}$ level that is due to $2 \bar{A} \rightarrow \bar{E}$ one-phonon spin-lattice relaxation.

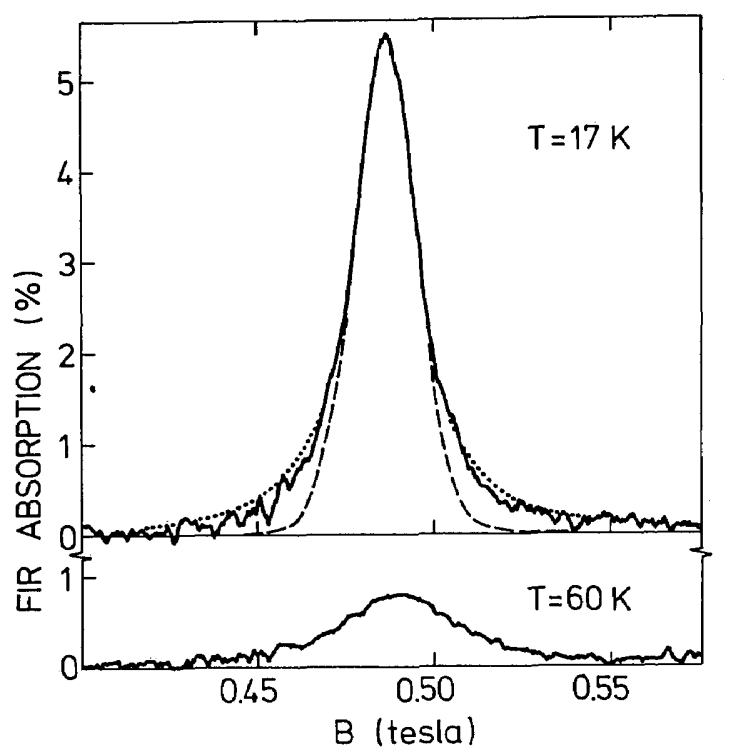

Fig. 2. Optically induced FIR absorption of excited chromium ions in ruby at two different temperatures. At low temperature the line profile is between a Gaussian (dashed line) and a Lorentzian (dotted line) profile; at high temperature it is Lorentzian.

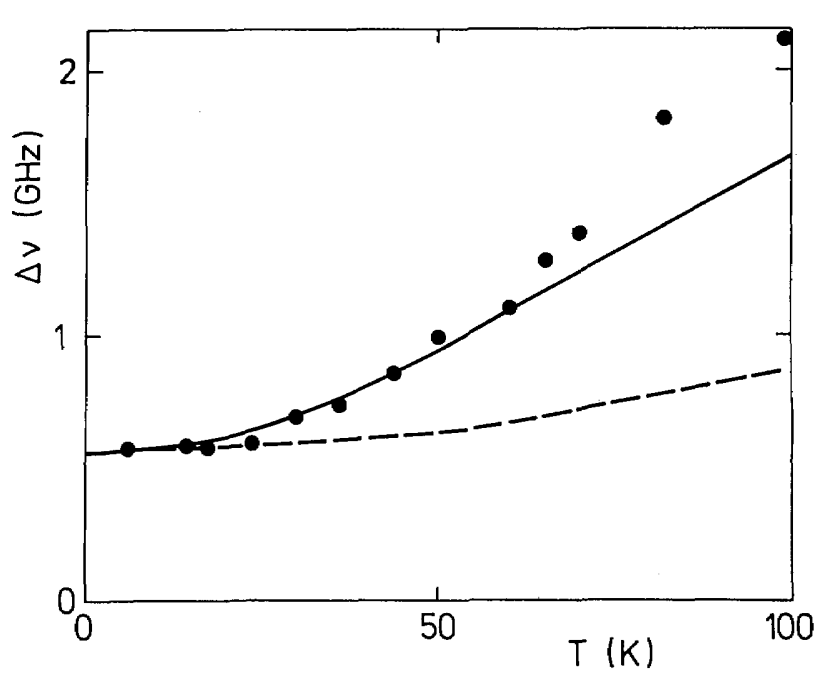

Fig. 3. Linewidths of the $\bar{E}_{-} \rightarrow 2 \bar{A}_{+}$absorption line of excited $\mathrm{Cr}^{3+}$ ions in ruby at different temperatures. The experimental values (filled circles) are best described by Eqs. (1) and (2) with $\Delta \nu_{0}=350 \mathrm{MHz}$ and $\Delta \nu_{G}=400 \mathrm{MHz}$ (solid curve). For comparison, a curve (dashed line) is shown for $\Delta \nu_{0}=150 \mathrm{MHz}$ and $\Delta \nu_{G}=450 \mathrm{MHz}$. 
For this process the temperature dependence of the homogeneous linewidth is given by the expression

$$
\Delta \nu_{L}=\Delta \nu_{0}(1+2 \bar{n}),
$$

where $\Delta \nu_{0}$ is the homogeneous linewidth at zero temperature and $\bar{n}$ is the thermal occupation number of $29-\mathrm{cm}^{-1}$ phonons stimulating the $2 \bar{A} \rightarrow \bar{E}$ relaxation processes. The thermal phonon occupation number is given by the Bose distribution $\bar{n}=[\exp (\Delta E / k T)$ $-1]^{-1}$.

The solid curve shown in Fig. 3 is obtained for $\Delta \nu_{0}=$ $350 \mathrm{MHz}$ and $\Delta \nu_{G}=400 \mathrm{MHz}$. With these values the experimental Voigt profile at low temperature can be described, and, moreover, the experimental temperature dependence is described well up to a crystal temperature of about $70 \mathrm{~K}$. Our results suggest therefore that $T_{1}=\left(2 \pi \Delta \nu_{0}\right)^{-1}=4.5 \times 10^{-10} \mathrm{sec}$, with an estimated error of $10 \%$. This value is in quite good agreement with the theoretical estimate ${ }^{1}$ already mentioned. With the value $\left(11 \times 10^{-10} \mathrm{sec}\right.$, with an estimated error of $\pm 30 \%$ ) reported by Rives and Meltzer, ${ }^{2}$ we would expect a homogeneous width $(150 \mathrm{MHz})$ that cannot describe the temperature dependence of the linewidth (dashed line in Fig. 3).

At temperatures above $70 \mathrm{~K}$ the experimental linewidth increases faster with increasing temperature than expected for a one-phonon process described by Eqs. (1) and (2). Our result indicates that an additional broadening mechanism occurs at high temperatures. We guess that the additional broadening is due to $2 \bar{A}$ $\rightarrow \bar{E}$ relaxation by phonon Raman processes. Assuming a $T^{7}$ dependence for the broadening, as expected for two-phonon Raman processes, the observed onset of the Raman process seems to be consistent with values of the linewidth of the $\bar{E} \rightarrow 2 \bar{A}$ transition measured at high temperatures (above $130 \mathrm{~K}$ ) by Raman spectroscopy. ${ }^{9}$

We note that in an earlier experiment on the same crystal FIR-induced fluorescence was studied ${ }^{10}$ and that it was concluded that the linewidth $\Delta \nu$ of the $\bar{E} \rightarrow 2 \bar{A}$ transitions at low temperature was smaller (by $30 \%$ ) than the value reported here. However, it was recently found that the apparent discrepancy is due to phonon bottleneck effects occurring in the fluorescence experiment. $^{3}$
It is known from measurements of the spin-lattice relaxation of the $\bar{E}$ state by Orbach processes through $2 \bar{A}$ states that the $2 \bar{A}_{ \pm} \rightarrow \bar{E}_{\mp}$ relaxation time has a value of $T_{+-}=15 \times 10^{-9}$ sec. $^{11}$ It follows therefore from our experiment that the $2 \bar{A}_{ \pm} \rightarrow \bar{E}_{ \pm}$relaxation time has a value of $T_{++}=T_{+-} T_{1}\left(T_{+-}+T_{1}\right)^{-1}=0.5 \times 10^{-9} \mathrm{sec}$ $( \pm 10 \%)$. The ratio $T_{++} / T_{+-}=30$ is in quite good agreement with the theoretical value of $50 .{ }^{1}$ Our experiment confirms, therefore, that for phonon transitions spin-flip transitions are much weaker than nonspin-flip transitions. The experiment shows, on the other hand, that for electromagnetic transitions, spinflip and non-spin-flip transitions have equal oscillator strengths. This indicates that these transitions are electric-dipole transitions. There exist, however, no calculations of the electric-dipole transition probabilities for the $\bar{E} \rightarrow 2 \bar{A}$ transitions in ruby.

We thank K. Lachner for technical assistance. The work was supported by the Deutsche Forschungsgemeinschaft.

\section{References}

1. M. Blume, R. Orbach, A. Kiel, and S. Geschwind, Phys. Rev. 139, A314 (1965).

2. J. E. Rives and R. S. Meltzer, Phys. Rev. B 16, 1808 (1977).

3. For a survey, see K. F. Renk and H. Lengfellner, in Proceedings of the International Conference on Lasers ' 80 , C. B. Collins, ed. (STS Press, McLean, Va., 1981), p. 398.

4. Delivered by Hrand Djevahirdjian Ltd., Monthey/Switzerland.

5. H. Lengfellner, U. Werling, J. Hummel, H. Netter, N. Retzer, and K. F. Renk, Solid State Commun. 38, 1215 (1981).

6. S. Geschwind, R. J. Collins, and A. L. Schawlow, Phys. Rev. Lett. 3, 545 (1959).

7. S. Sugano and I. Tsujikawa, J. Phys. Soc. Jpn. 13, 899 (1958).

8. D. W. Posener, Austral. J. Phys. 12, 184 (1959).

9. B. Halperin, J. A. Koningstein, and D. Nicollin, Chem. Phys. Lett. 68, 58 (1979).

10. H. Lengfellner, G. Pauli, W. Heisel, and K. F. Renk, Appl. Phys. Lett. 29, 566 (1976).

11. S. Geschwind, G. E. Devlin, R. L. Cohen, and S. R. Chinn, Phys. Rev. A 137, 1087 (1965). 